\title{
MODELS OF SPACE-TIME
}

\author{
BY W. F. EBERLEIN ${ }^{1}$
}

Communicated by M. Kac, May 17, 1965

1. Introduction. In [1] we exhibited electron spin as a nonrelativistic geometric property of (a model of) Euclidean 3-space. We now extend our model to one of space-time. The connections between 2 and 4 component spinors become lucid, while the Dirac equation and its relativistic "invariance" properties undergo a fundamental simplification and clarification.

2. Abstract space-time. We need first an axiomatic foundation strong enough to support both our mathematical considerations and their applications to physics.

Definition. An $n+1$ dimensional space-time $(n \geqq 1)$ consists of

(A) An $n+1$ dimensional vector space $V$ over the real numbers plus a symmetric bilinear real form $A \cdot B$ (inner product) such that:

(1) There exists a vector $A$ with $A \cdot A<0$.

(2) Any 2-dimensional subspace of $V$ contains a vector $A$ with $A \cdot A>0$.

(B) A set $\chi$ of objects $p, q, \cdots$ (points or "events") plus a mapping $(p, q) \rightarrow p-q$ of $\chi \times \chi$ into $V$ such that:

(1) $(p-q)+(q-r)=p-r$.

(2) $p-q=0$ implies $p=q$.

(3) Given any point $q$ and any vector $A$ there exists a point $p$ with $p-q=A$.

Any $V$ satisfying (A) yields a model of space-time (vector spacetime) on setting $\chi=V$. The Minkowski model $V=\chi=R_{M}^{n+1}$ consists of all $n+1$-tuples of real numbers $x=\left(x_{1}, \cdots, x_{n}, x_{n+1}\right)$ with $x \cdot y$ $=x_{1} y_{1}+\cdots+x_{n} y_{n}-x_{n+1} y_{n+1}$. (When $n=3, x_{4}=c t$, where $t$ is time and $c$ is the velocity of light.) Every $n+1$ dimensional vector spacetime is isomorphic to $R_{M}^{n+1}$, but this result is physically misleading. Eventually we set $n=3, \chi=$ the physical space-time continuum, and $V=\mathfrak{E}_{4}$, the spin model of (vector) space-time we shall construct.

3. The models $\mathbb{E}_{3}$ and $W_{4}$. In [1] we defined the spin model $\mathbb{E}_{3}$ of Euclidean 3-space as the vector space of self-adjoint linear transformations of trace 0 in a 2-dimensional unitary space $H_{2}$ (spin space) plus the operations $A \cdot B=(1 / 2)(A B+B A)$ and $A \times B=(1 / 2 i)$

1 Work supported by National Science Foundation Grant NSF G-17774. 
- $(A B-B A)$. (We identify a scalar $c$ with $c I$, where $I$ is the identity transformation in $H_{2}$.) In general we denote the algebra of linear transformations in a vector space $E$ by $B(E)$. We summarize some results of [1] that we need:

Relative to an arbitrary orthonormal basis $\phi_{1}, \phi_{2}$ for $H_{2}$ any vector $A$ in $E_{3}$ has the matrix representation

$$
A \underset{(\phi)}{\stackrel{\leftrightarrow}{\longrightarrow}}\left(\begin{array}{cc}
x_{3} & x_{1}-i x_{2} \\
x_{1}+i x_{2} & -x_{3}
\end{array}\right)=x_{1} \sigma_{1}+x_{2} \sigma_{2}+x_{3} \sigma_{3},
$$

where

$$
\sigma_{1}=\left(\begin{array}{ll}
0 & 1 \\
1 & 0
\end{array}\right), \quad \sigma_{2}=\left(\begin{array}{rr}
0 & -i \\
i & 0
\end{array}\right), \quad \sigma_{3}=\left(\begin{array}{rr}
1 & 0 \\
0 & -1
\end{array}\right),
$$

are the Pauli matrices. Then $\mathbb{E}_{3}$ is 3-dimensional and

$$
A \cdot A=A^{2}=x_{1}^{2}+x_{2}^{2}+x_{3}^{2}=-\operatorname{det} A .
$$

Let $\mathrm{SU}(2)$ denote the group of unitary transformations in $H_{2}$ of determinant 1 and $\mathrm{SO}(3)$, the group of rotations or orthogonal transformations of determinant 1 in $\mathbb{E}_{3}$. Given $U$ in $\mathrm{SU}(2)$ set $R_{U} A$ $=U A U^{-1}\left(A \in \mathbb{F}_{3}\right)$. Then $R_{U}$ is a linear transformation in $\mathfrak{E}_{3}$, and the mapping $U \rightarrow R_{U}$ is a 2-to-1 homomorphism of SU(2) onto $\mathrm{SO}(3)$.

The obvious extension of $E_{3}$ is the vector space $W_{4}$ consisting of all self-adjoint linear transformations in $H_{2}$. Then for any $A$ in $W_{4}$

$$
A \underset{(\phi)}{\stackrel{\leftrightarrow}{\longrightarrow}}\left(\begin{array}{cc}
x_{4}+x_{3} & x_{1}-i x_{2} \\
x_{1}+i x_{2} & x_{4}-x_{3}
\end{array}\right)=x_{1} \sigma_{1}+x_{2} \sigma_{2}+x_{3} \sigma_{3}+x_{4}
$$

and $-\operatorname{det} A=x_{1}^{2}+x_{2}^{2}+x_{3}^{2}-x_{4}^{2} \equiv A \cdot A . W_{4}$ is then a $3+1$-dimensional vector space-time, but the corresponding inner product is hybrid:

$$
A \cdot B=\frac{1}{2}(A B+B A)-\frac{1}{2}(\operatorname{trace} B) A-\frac{1}{2}(\operatorname{trace} A) B .
$$

One can now extend the covering map above by setting $\operatorname{SL}(2, C)$ $=$ the group of linear transformations in $H_{2}$ of determinant $1, \&+$ $=$ the homogeneous proper orthochronous Lorentz group; i.e., the linear transformations in $W_{4}$ that preserve the inner product, have determinant 1 , and don't exchange past and future. Given $S$ in $\operatorname{SL}(2, C)$ set $M_{S} A=S A S^{*}\left(A \in W_{4}\right)$. Then $M_{S}$ is a linear transformation in $W_{4}$, and one has the extended

TheOREM 3.1. The mapping $S \rightarrow M_{S}$ is a 2-to-1 homomorphism of $\mathrm{SL}(2, C)$ onto \&f.

This result is essentially known in matrix disguise, but the co- 
ordinate-free methods of [1] afford a simpler and more incisive proof than is to be found in the literature.

Although its inner product lacks the Jordan form substituting in $E_{3}$, the model $W_{4}$ is appropriate to analysis of the Maxwell equations and the Weyl neutrino, as we shall show in a later paper.

4. The antiquaternion unit $J$. What one wants is an element $J$ in $B\left(H_{2}\right)$ with real square and anticommuting with $\mathcal{E}_{3}$. But the only element of $B\left(H_{2}\right)$ that anticommutes with $E_{3}$ is 0 . For the same reason no nonsingular $U$ in $B\left(H_{2}\right)$ yields the space inversion $P: R_{U} A=U A U^{-1}$ $=-A\left(A \in \mathbb{E}_{3}\right)$. We are thus led to the following

PROBLEM. Find all antilinear transformations $J$ in $H_{2}$ anticommuting with $\mathfrak{E}_{3}$, in particular those such that $J^{2}= \pm 1$.

In an equivalent guise (commutativity of $J$ with the quaternion algebra $Q=[k U: k \geqq 0, U \in \mathrm{SU}(2)]$ (cf. [2])) we obtained in [4] the following

Solution. Given an arbitrary orthonormal basis $\phi_{1}, \phi_{2}$ in $H_{2}$, identify a vector $x_{1} \phi_{1}+x_{2} \phi_{2}$ with the column vector

$$
\left(\begin{array}{l}
x_{1} \\
x_{2}
\end{array}\right)
$$

Then every such $J$ is of the form

$$
\left(\begin{array}{l}
x_{1}^{\prime} \\
x_{2}^{\prime}
\end{array}\right)=J\left(\begin{array}{l}
x_{1} \\
x_{2}
\end{array}\right)=\omega\left(\begin{array}{c}
-\bar{x}_{2} \\
\bar{x}_{1}
\end{array}\right)=\omega\left(\begin{array}{rr}
0 & -1 \\
1 & 0
\end{array}\right)\left(\begin{array}{l}
\bar{x}_{1} \\
\bar{x}_{2}
\end{array}\right),
$$

whence $J^{2}=-|\omega|^{2} \neq 1$ and $J^{2}=-1$ iff $|\omega|=1$-i.e., iff $J$ is antiunitary.

The normalized $J, J^{2}=-1$, thus obtained is unique up to a phase factor and may be identified with Wigner's nonrelativistic time-inversion operator for particles of spin $\frac{1}{2}$, but the idea goes back to Möbius: The space inversion operator $R_{J} A=J A J^{-1}=-A\left(A \in \mathcal{E}_{3}\right)$ arising is independent of the scalar $\omega \neq 0$, whence one can regard (1) as an antiprojective transformation in homogeneous coordinates. Set $z=x_{1} / x_{2}$, $z^{\prime}=x_{1}^{\prime} / x_{2}^{\prime}$ to obtain

$$
z^{\prime}=-\bar{z}^{-1}
$$

Now map onto the Riemann sphere, $z \rightarrow \xi$, and note that $\xi^{\prime}$ is antipodal to $\xi$.

We can now rewrite the defining properties of $\mathfrak{E}_{3}$ as follows:

$\mathcal{E}_{3}$ consists of all $T$ in $B\left(H_{2}\right)$ such that

$$
i T=T^{*} i, \quad J T=-T^{*} J
$$


while the identity $A^{*} J A=(\operatorname{det} A)^{-} J$ for $A$ in $B\left(H_{2}\right)$ translates the defining properties of $\mathrm{SU}(2)$ into:

$\mathrm{SU}(2)$ consists of all $\mathrm{T}$ in $B\left(\mathrm{H}_{2}\right)$ such that

$$
\begin{aligned}
& T^{*} i T=i, \\
& T^{*} J T=J .
\end{aligned}
$$

These formulae are independent of the phase factor for the normalized $J$. We now pick a distinguished $J$. This amounts to putting a complex orientation on $\mathrm{H}_{2}$ (cf. [4]).

5. The spin model $\mathbb{E}_{4}$ and the group $\mathcal{G}_{+}^{\uparrow}$. Now let $E_{4}$ be $H_{2}$ considered as a real vector space plus the new inner product

$$
\langle x \mid y\rangle_{+}=R(\langle x \mid y\rangle) \text {. }
$$

$E_{4}$ is a 4 -dimensional Euclidean vector space. Linear and antilinear transformations in $\mathrm{H}_{2}$ are then on the same footing as linear transformations in $\mathbb{E}_{4}$, betraying their origin only in commutativity or anticommutativity with the now distinguished linear transformation i. $S=T^{*}$ in $B\left(H_{2}\right)$ implies $S=T^{*}$ in $B\left(E_{4}\right)$, while the new and old trace and determinant of a $T$ from $B\left(H_{2}\right)$ are connected as follows:

$$
\begin{aligned}
\operatorname{trace}_{4} T & =2 R\left(\operatorname{trace}_{2} T\right), \\
\operatorname{det}_{4} T & =\left|\operatorname{det}_{2} T\right|^{2} .
\end{aligned}
$$

DEFINITION. $\mathbb{E}_{4}$ consists of all linear transformations in $E_{4}$ satisfying (3).

Clearly $\xi_{4}$ is a subspace of $B\left(E_{4}\right)$ containing $\xi_{3}$ and closed under *.

ThEOREM 5.1. $\mathfrak{F}_{4}$ consists of all elements of $B\left(E_{4}\right)$ of the form

$$
T=A+a J\left(A \in \mathfrak{E}_{3}, a \text { real }\right) .
$$

Then $T^{2}=A^{2}-a^{2}$ and we can set $T_{1} \cdot T_{2}=\frac{1}{2}\left(T_{1} T_{2}+T_{2} T_{1}\right)$ to obtain a $3+1$ dimensional model of vector space-time.

Let $K=(1+J) / 2^{1 / 2}$. Then $K$ is orthogonal, $K^{2}=J$, and $K^{8}=1$.

Theorem 5.2. The mapping $\tau: A \rightarrow K A K$ is an isomorphism of $W_{4}$ onto $\mathbb{E}_{4}$ leaving $\mathbb{E}_{3}$ pointwise fixed and preserving the inner product.

Since every $T$ in $B\left(E_{4}\right)$ admits a unique decomposition $T=T_{1}+T_{2}$, where $T_{1}, T_{2}$ are respectively linear and antilinear transformations in $H_{2}$, the space-time $\mathfrak{E}_{4}$ splits naturally into space and time.

DEFINITION. $\mathcal{G}_{+}^{\uparrow}$ consists of all linear transformations $T$ in $E_{4}$ satisfying (4).

THEOREM 5.3. $\mathcal{G}_{+}^{\uparrow}$ is a group containing $\mathrm{SU}(2)$ and closed under *. 
If $T \in \mathcal{G}_{+}^{\uparrow}$ set $L_{T} A=T A T^{-1}\left(A \in \mathbb{E}_{4}\right)$. Then $L_{T}$ is a linear transformation in $\mathbb{E}_{4}$, and

THEOREM 5.4. The mapping $T \rightarrow L_{T}$ is a 2-to-1 homomorphism of $\mathcal{S}_{+}^{\uparrow}$ onto $\mathcal{L}_{+}^{\uparrow}$.

Space-inversion $P$ and time-reversal $T$ arise as follows: $P$ : $A \rightarrow J A J^{-1}, T: A \rightarrow i A i^{-1}$. Let $\mathrm{g}$ be the group of linear transformations in $E_{4}$ generated by $\mathcal{S}_{+}^{\uparrow}, J$, and $i$.

The connection between 2- and 4-component spinors is then contained in

THEOREM 5.5. The mapping $\nu: S \rightarrow K S K^{-1}$ is an isomorphism of $\mathrm{SL}(2, C)$ onto $\mathcal{G}_{+}^{\uparrow}$ leaving $\mathrm{SU}(2)$ pointwise fixed.

THEOREM 5.6. The following diagram is commutative:

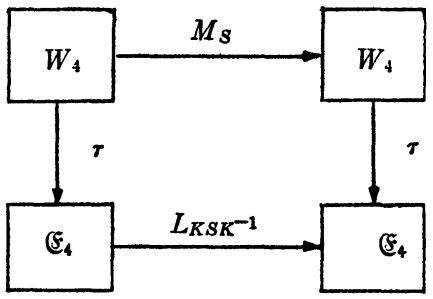

Note that $\operatorname{det}_{4} K S K^{-1}=\operatorname{det}_{4} S=\left|\operatorname{det}_{2} S\right|^{2}=1$, while $\operatorname{det} J=\operatorname{det} K$ $=1$ and $\operatorname{det}_{4} i=1$, whence $\mathcal{G}_{+}^{\uparrow}$ (or $\mathcal{G}$ ) and $\operatorname{SL}(2, C)$ are subgroups of $\mathrm{SL}(4, R)$ whose intersection is $\mathrm{SU}(2)$.

$\mathbb{E}_{4}$ is also remarkable in that it admits an explicit coordinate-free oriented volume function $\theta\left(A_{1}, A_{2}, A_{3}, A_{4}\right)=\frac{1}{4}$ trace $_{4}\left(i A_{1} A_{2} A_{3} A_{4} J\right)$, reducing to $(1 / 2 i)$ trace $\left(A_{1} A_{2} A_{3}\right)=\left(A_{1} \times A_{2}\right) \cdot A_{3}$ when $A_{4}=J$ and $A_{1}, A_{2}, A_{3}$ lie in $\mathcal{E}_{3}$ (cf. [3]). Finally, the (Clifford) algebra generated by $\mathbb{E}_{4}$ is just $B\left(E_{4}\right)$.

6. The Dirac operator. Let $\left(g_{i j}\right)=\operatorname{diag}(1,1,1,-1)$. Then an ordered orthonormal basis $(e)$ for $\xi_{4}$ is characterized by the identity

$$
e_{i} e_{j}+e_{j} e_{i}=2 g_{i j} \text {. }
$$

Let $E_{4}^{c}$ and $\mathfrak{E}_{4}^{c}$ be the respective complexifications of $E_{4}$ and $\mathfrak{E}_{4}$ and consider the expression $\langle A u, v\rangle$, where $A$ runs over $\xi_{4}$ and $u, v$ run over $E_{4}^{c}$. Since this expression is real linear in $A$, complex linear in $u$, and complex antilinear in $v$, there exists a unique mapping $F: E_{4}^{c}$ $\times E_{4}^{c} \rightarrow \mathbb{E}_{4}^{c}$ such that

$$
\langle A u \mid v\rangle=A \cdot F(u, v),
$$


and $F(u, v)$ is complex linear in $u$ and complex antilinear in $v$. In particular, $F(u, J u)$ lies in $\mathbb{E}_{4}$.

Given now any ordered o.n. basis $e_{1}, \cdots, e_{4}$ for $E_{4}$ consider smooth functions $\psi: \xi_{4} \rightarrow E_{4}^{c}$ and let

$$
\left(\partial_{j} \psi\right)(x)=\lim _{h \rightarrow 0} \frac{\psi\left(x+h e_{j}\right)-\psi(x)}{h} .
$$

Definition. The Dirac operator $D=e_{1} \partial_{1}+e_{2} \partial_{2}+e_{3} \partial_{3}-e_{4} \partial_{4}$. Then $D^{2}=\partial_{1}^{2}+\partial_{2}^{2}+\partial_{3}^{2}-\partial_{4}^{2}$, the d'Alembertian, while the Dirac equation takes the form

$$
D \psi+\kappa \psi=0 \quad(\kappa=m c / \hbar),
$$

and the associated charge-current vector $-F(\psi, J \psi)$ satisfies the continuity equation

$$
\operatorname{div} F(\psi, J \psi)=0 .
$$

Finally the relativistic "invariance" properties of the Dirac equation reduce to simple properties of the Dirac operator $D$.

Theorem 6.1 (Passive invariance). $\langle D \psi \mid u\rangle=\operatorname{div} F(\psi, u)\left(u \in E_{4}^{c}\right)$.

If $T \in \mathcal{g}$, let $(\hat{T} \psi)(x)=T \psi\left(L_{T^{-1}} x\right)=T \psi\left(T^{-1} x T\right)$.

Theorem 6.2 (ACtive invariance). $D \hat{T}=\hat{T} D$.

Proofs of the above theorems and some related results will appear elsewhere.

\section{REFERENCES}

1. W. F. Eberlein, The spin model of Euclidean 3-space, Amer. Math. Monthly 69 (1962), 587-598.

2. - The geometric theory of quaternions, Amer. Math. Monthly 70 (1963), 952-954.

3. - Vector identities in $\mathfrak{E}_{8}$, Amer. Math. Monthly 71 (1964), 302.

4. - Axiomatic charge conjugation, J. Math. Phys. (to appear).

UNIVERSITY OF ROCHESTER 